\title{
Composición Corporal y Estado Nutricional de una Muestra de Estudiantes de 9 a 12 Años de Edad de Colegios Municipalizados de la Comuna de Padre las Casas, Región de la Araucanía-Chile
}

\author{
Body Composition and Nutritional Status of 9 to 12 Year Old Municipal Schools \\ Students Sample of Padre las Casas Commune, Araucanía Region, Chile
}

Cristián Martínez*; Otto Reinike*; Héctor Silva**; Vanessa Carrasco*; Erika Collipal** \& Carlos Jiménez****

\begin{abstract}
MARTÍNEZ, C.; REINIKE, O.; SILVA, H.; CARRASCO, V.; COLLIPAL, E. \& JIMÉNEZ, C. Composición Corporal y Estado Nutricional de una Muestra de Estudiantes de 9 a 12 años de edad de Colegios Municipalizados de la Comuna de Padre las Casas, Región de la Araucanía-Chile. Int. J. Morphol., 31(2):425-431, 2013.
\end{abstract}

RESUMEN: El estudio del Índice de Masa Corporal (IMC), es el parámetro recomendado por la OMS para conocer el estado nutricional de acuerdo a la norma técnica nutricional vigente en Chile. Los estudios antropométricos permiten estimar la composición corporal, estudiar la morfología y dimensiones. Este estudio describe la realidad nutricional y la composición corporal de una muestra de los colegios municipalizados de la comuna de Padre las Casas. Evaluamos 122 adolescentes de ambos sexos, 65 hombres (53,2\%) y 57 mujeres (46,7\%), entre 9 y 12 años de edad. Para obtener la composición corporal se utilizó el método antropométrico descrito por Dhebora Kerr, mientras que la evaluación y clasificación del estado nutricional se realizo de acuerdo a las normas técnica nutricional del Ministerio de Salud de Chile. Los hombres presentaron una composición corporal donde predomina la masa muscular (MM) sobre los otros componentes, con valores de $38,79 \pm 3,65 \%$ a los 9 años de edad, $40,80 \pm 3,36 \%$ a los 10 años de edad, $41,47 \pm 2,52 \%$ a los 11 años de edad, $43,35 \pm 1,59 \%$ a los 12

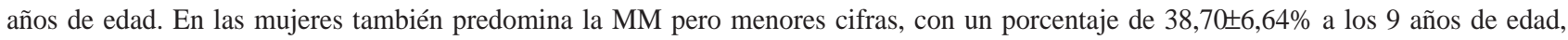
$39,85 \pm 3,84 \%$ a los 10 años de edad, 39,56 $\pm 4,41 \%$ a los 11 años de edad, 40,28 $\pm 2,37 \%$ a los 12 años de edad. El estado nutricional de la muestra tiene los siguientes resultados en el caso de los varones el 23,1\% fueron obesos y el 35,4\% presentaron sobrepeso. En las damas el 26,3\% fueron obesas y un 15,8\% presentaron sobrepeso. Al comparar los resultados de Masa Adiposa y MM se encontraron diferencias significativas por género y entre las edades de la muestra con un valor de $p \leq 0,05$. El ambiente obesigénico en donde se destaca la modificación del estilo de vida, la alimentación y el efecto del sedentarismo, está afectando a la población escolar de Padre Las Casas.

PALABRAS CLAVE: Antropometría; Índice de masa corporal; Estado Nutricional.

\section{INTRODUCCIÓN}

Estudios de la composición corporal por método pentacompartimental y el Îndice de Masa Corporal (IMC) de una población determinada permite conocer su estado nutricional y su morfoestructura. La OMS (2009), define la obesidad como una enfermedad crónica, multifactorial, con numerosas complicaciones, la cual es el resultado de la unión de factores biológicos, genéticos y ambientales. Los factores más estudiados de la obesidad están relacionados con los estilos de vida, actividad física, nivel socioeconómico, el género, y la edad, siendo por lo tanto, una enfermedad compleja y dinámica (Peña \& Bacallao, 2006).
En nuestro país, el Ministerio de Salud en el año 2003 propuso utilizar el IMC por edad como criterio de evaluación nutricional en escolares y adolescentes (MINSAL, 2003). El IMC es considerado el mejor indicador antropométrico para diagnosticar el sobrepeso y la obesidad en niños entre los 2 y los 18 años (Burrows et al., 2004).

Un estudio reciente efectuado en una muestra nacional de escolares de $8^{\circ}$ básico de edades entre 13-15 años encontró que el 40,2\% de ellos presentaban sobrepeso u

\footnotetext{
Departamento de Educación Física, Universidad de La Frontera, Temuco, Chile.

** Departamento de Ciencias Básicas, Universidad de La Frontera, Temuco, Chile.

**** Departamento de Matemática y Estadística, Universidad de La Frontera, Temuco, Chile.

Proyecto DIUFRO N 080059 Dirección de Investigación Universidad de La Frontera, Temuco, Chile.
} 
MARTíneZ, C.; REINIKE, O.; SILVA, H.; CARRASCO, V.; COLLIPAL, E. \& JIMÉNEZ, C. Composición Corporal y Estado Nutricional de una Muestra de Estudiantes de 9 a 12 años de edad de Colegios Municipalizados de la Comuna de Padre las Casas, Región de la Araucanía-Chile. Int. J. Morphol., 31(2):425-431, 2013.

obesidad (MINEDUC, 2010). Lo más preocupante es que la condición física global de esta misma muestra se encontró deficiente en el 90,8\% de los casos. Esta información no existe para otros grupos de edad escolar. Coincidentemente, datos existentes en adultos provenientes de 5 Regiones del país incluida la Región de La Araucanía, muestran que la situación es deficiente en la condición física estuvo presente en el 84,5\% de los casos (Díaz et al., 2008). En la encuesta de salud del año 2010, la prevalencia de exceso de peso en la población adulta es del $64,5 \%$, la cual corresponde al 39,32\% de sobrepeso y un $25,13 \%$ de obesidad (MINSAL, 2011). Entre otros riesgos, del sobrepeso y la obesidad en adolescentes, es el incrementó de padecer enfermedades a las arterias coronarias y síndrome metabólico en edad adulta (Alegría et al., 2005; Bibbins-Domingo et al., 2007).

La antropometría tiene como finalidad conocer las características morfológicas de una población. En este estudio hemos utilizado el método de fraccionamiento corporal de Dhebora Kerr (Kerr, 1988), validado por el estudio de Bruselas de disección de cadáveres (Clarys et al., 1984) tanto para pesos estructurados y obtenidos para las distintas masas corporales. Este es un método pentacompartimental, que divide la morfoestructura en: masa adiposa (MA), masa muscular (MM), masa ósea (MO), masa residual (MR) y masa de la piel (MP) (Almagià et al., 2009).

Los estudios antropométricos permiten estimar la composición corporal, estudiar la morfología, dimensiones y proporcionalidad en relación al rendimiento deportivo, nutrición y crecimiento (Carter \& Heath-Roll, 1990; Cheng-Ye \& Ohsawa, 1996; Sánchez-Muñoz et al., 2007; Álvero et al., 2009).

El análisis de los pliegues cutáneos permite estimar el contenido de masa grasa y masa libre de grasa en los diferentes individuos y consecuentemente, inferir los requerimientos nutricionales (Toro \& Almagià, 1989).

Se han desarrollado estudios antropométricos, en población deportiva, de jóvenes físicamente activos (Almagià et al., 2008; Almagià et al., 2009; da Silva, 2011) en etnias Aymara, Mapuches (del Sol \& Henríquez, 1987; Espinoza et al., 2009) encontrándose diferentes resultados de acuerdo a la población estudiada.

En Chile se manifiesta un aumento de la obesidad a medida que aumenta la edad escolar, con diferencias marcadas en el género femenino, similares resultados se han observado en los estudios realizados con muestras de niños y adolescentes de colegios municipalizados urbanos y rurales de la Región de la Araucanía (Silva et al., 2003, 2005, 2008; Martínez et al., 2008; Carrasco et al., 2011).

Por tanto, el objetivo de este estudio es analizar la composición corporal mediante un método pentacompartimental y determinar el estado nutricional en una muestra de estudiantes de 9 a 12 años de establecimientos municipalizados de la comuna de Padre las Casas Región de la Araucanía - Chile

\section{MATERIAL Y METODO}

Para este estudio de composición y del IMC, se evaluaron 122 sujetos de ambos sexos, 67 hombres $(54,9 \%)$ y 55 mujeres $(45,1 \%)$, entre 9 y 12 años de edad, que cumplieron los criterios de inclusión. Para obtener la composición corporal se utilizó el método antropométrico descrito por Débora Kerr en el año 1988 que consiste en el fraccionamiento corporal en cinco componentes masa muscular, masa ósea, masa adiposa, masa piel y masa residual, mientras que la evaluación y clasificación del estado nutricional se realizo de acuerdo a las normas técnicas nutricionales.

Los datos fueron registrados en una ficha antropométrica de papel donde se registró el peso. Para la medición del peso corporal, se empleó una balanza digital marca Tanita modelo UM 2204 precisión de 0,2 kilos con una capacidad máxima de 136 kilos. La talla sentada fue medida usando un estadiómetro de pared, graduado en $\mathrm{mm}$. Con antropómetro y cinta antropométrica de metal marca Rosscraft se evaluaron los perímetros de cabeza, brazo relajado, brazo flexionado en tensión, antebrazo máximo, tórax mesoesternal, cintura mínima, cadera máximo, muslo máximo, muslo medial pantorrilla máxima, y los diámetros biacromial, tórax transverso, tórax anteroposterior, biiliocrestídeo, humeral y femoral. Con un adipómetro de marca Harpenden, se midieron los siguientes pliegues tríceps, bíceps, subescapular, cresta ilíaca, supraespinal, abdominal, muslo frontal y pantorrilla máxima. Luego se digitaron los datos obtenidos en el programa Antropogym. La evaluación se hizo empleando técnicas y estándares descritos por la Sociedad Internacional para el avance de la Kinantropometría (ISAK, 2001).

El IMC se calculó a través de la formula Peso $(\mathrm{kg}) /$ Talla (m)2 usando la clasificación propuesta en la norma técnica de evaluación nutricional de niños y niñas de 6 a 18 años del Ministerio de Salud de Chile (MINSAL, 2004). Según este indicador se clasificó como sigue: bajo peso, menor al percentil (p) 10, normal; entre p10-p85, sobrepeso; entre p85-p95, obesidad; sobre el p95. 
MARTínEZ, C.; REINIKE, O.; SILVA, H.; CARRASCO, V.; COLLIPAL, E. \& JIMÉNEZ, C. Composición Corporal y Estado Nutricional de una Muestra de Estudiantes de 9 a 12 años de edad de Colegios Municipalizados de la Comuna de Padre las Casas, Región de la Araucanía-Chile. Int. J. Morphol., 31(2):425-431, 2013.

Para el procesamiento y análisis de los datos se utilizó el programa estadístico SPSS versión 17.0. Dependiendo del comportamiento de los datos, se utilizaron Test paramétricos y no paramétricos con estadísticos descriptivos y estableciendo las posibles diferencias significativas entre las variables de Masa Muscular (MM) y Masa Adiposa (MA) por sexo y entre las diferentes edades de los grupos, mediante las pruebas estadísticas, Kruskal- Wallis, T Student y U de Mann - Whitney. Se empleó un error de a $\leq 0,05$ para establecer el nivel de significancia

\section{RESULTADOS}

La composición corporal en adolecentes de ambos sexos según edad de Padre las Casas -Chile, pueden ser observados en las Tablas I y II.

En la Tabla III se expresan los valores y porcentajes del estado nutricional de ambos separados por edad y sexo con sus respectivos porcentajes del estado nutricional de la muestra

Tabla I. Presenta los valores de tendencia central y dispersión de la muestra, valores mínimos, y máximos de los cinco componentes de la composición corporal de los hombres pertenecientes a las escuelas municipalizadas de la comuna de Padre las Casas Región de la Araucanía Chile ( $\mathrm{n}=65)$.

\begin{tabular}{|c|c|c|c|c|c|}
\hline \multirow{5}{*}{ Masa Adiposa } & & *9 años & *10 años & *11 años & 12 años \\
\hline & Promedio ** & 27,44 & 28,29 & 27,73 & 24,66 \\
\hline & DE & 5,05 & 5,085 & 4,61 & 3,00 \\
\hline & Mínimo & 19,68 & 19,29 & 18,37 & 20,88 \\
\hline & Máximo & 36,28 & 35,52 & 35,57 & 31,53 \\
\hline \multirow[t]{4}{*}{ Masa Muscular } & Promedio * & 38,79 & 40,80 & 41,47 & 43,35 \\
\hline & DE & 3,65 & 3,366 & 2,52 & 1,59 \\
\hline & Mínimo & 32,39 & 35,66 & 36,02 & 40,01 \\
\hline & Máximo & 45,24 & 44,70 & 45,66 & 46,24 \\
\hline \multirow[t]{4}{*}{ Masa Residual } & Promedio & 13,89 & 11,77 & 12,34 & 12,42 \\
\hline & $\mathrm{DE}$ & 2,91 & 1,13 & 1,27 & 0,82 \\
\hline & Mínimo & 11,52 & 10,66 & 10,36 & 10,89 \\
\hline & Máximo & 23,34 & 14,91 & 15,29 & 13,60 \\
\hline \multirow[t]{4}{*}{ Masa Ós ea } & Promedio & 13,08 & 12,76 & 12,62 & 13,28 \\
\hline & DE & 1,24 & 1,08 & 1,48 & 0,99 \\
\hline & Mínimo & 11,63 & 11,26 & 10,54 & 11,10 \\
\hline & Máximo & 15,76 & 14,42 & 15,58 & 15,12 \\
\hline \multirow[t]{4}{*}{ Masa Piel } & Promedio & 6,77 & 6,35 & 5,82 & 6,27 \\
\hline & $\mathrm{DE}$ & 1,24 & 0,83 & 0,92 & 0,72 \\
\hline & Mínimo & 4,94 & 5,31 & 4,39 & 5,37 \\
\hline & Máximo & 8,69 & 7,85 & 7,92 & 7,81 \\
\hline
\end{tabular}

* Significativo $\mathrm{P} \leq 0,05 \quad{ }^{* *}$ No significativo $\mathrm{P}>0,05$

\section{DISCUSIÓN}

Los resultados encontrados permiten comparar la muestra de estudiantes municipalizados de la comuna de Padre las Casas, con otros estudios regionales. Los cuales nos indican que se mantiene la tendencia de la obesidad, que dice que la población femenina presenta un mayor porcentaje de obesidad, $26,3 \%$, comparado con los varones 
MARTíneZ, C.; REINIKE, O.; SILVA, H.; CARRASCO, V.; COLLIPAL, E. \& JIMÉNEZ, C. Composición Corporal y Estado Nutricional de una Muestra de Estudiantes de 9 a 12 años de edad de Colegios Municipalizados de la Comuna de Padre las Casas, Región de la Araucanía-Chile. Int. J. Morphol., 31(2):425-431, 2013.

Tabla II. Presenta los valores de tendencia central y dispersión de la muestra, valores mínimos y máximos de los cinco componentes de la composición corporal de las mujeres pertenecientes a las escuelas municipalizadas de la comuna de Padre las Casas. Región de la AraucaníaChile ( $\mathrm{n}=57)$.

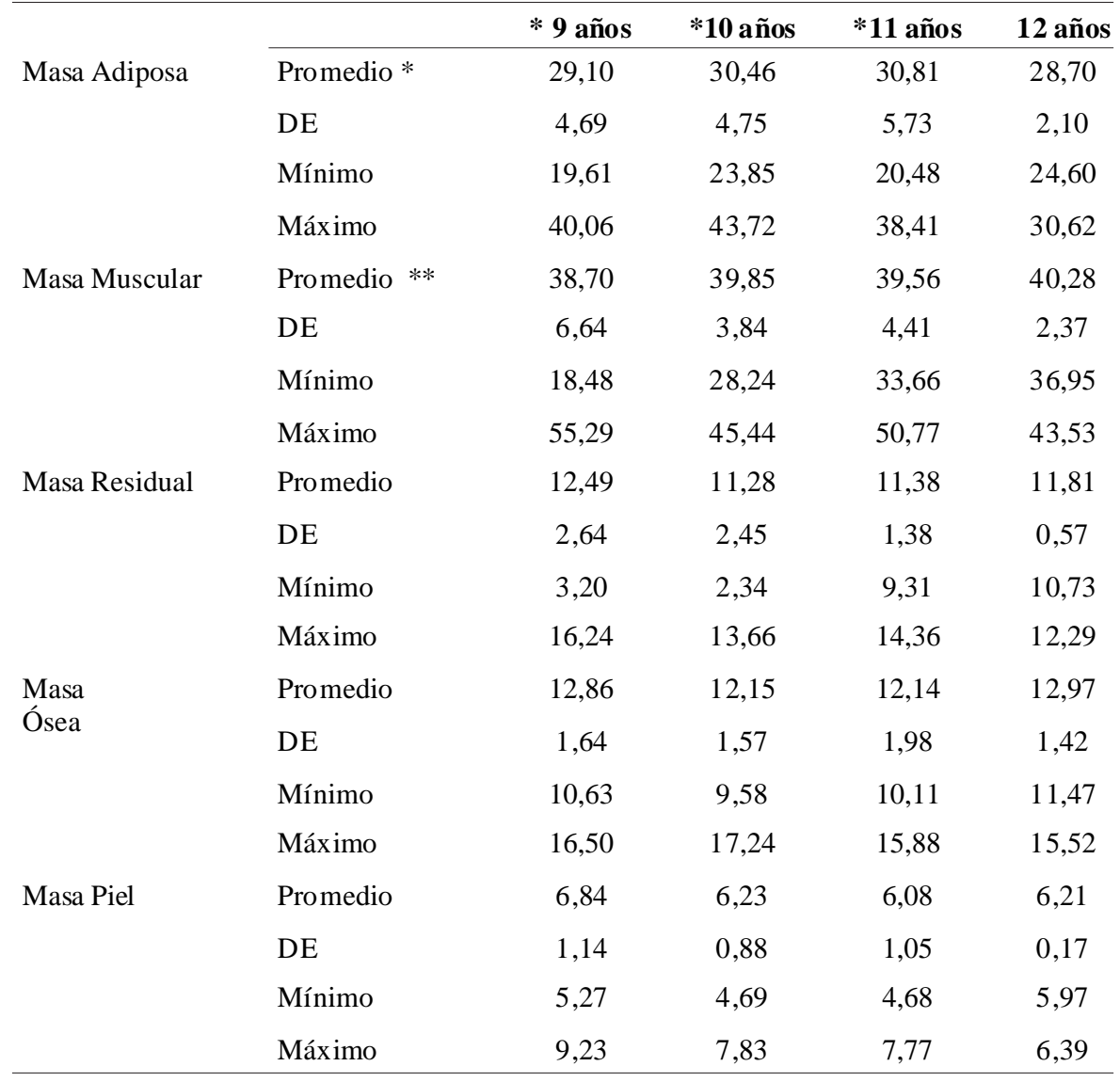

* Significativo $\mathrm{P}>0,05$. ** No significativo $\mathrm{P} \leq 0,05$

Tabla III. Diagnóstico Nutricional de una muestra de adolescentes de 9 a 12 años de ambos sexos de la Comuna de Padre las Casas, Región de la Araucanía - Chile $(n=122)$.

\begin{tabular}{lcccccc}
\hline & \multicolumn{2}{c}{ Hombres } & \multicolumn{2}{c}{ Mujeres } & \multicolumn{2}{c}{ Total } \\
\cline { 2 - 7 } & $\mathbf{n}$ & $\boldsymbol{\%}$ & $\mathbf{n}$ & $\boldsymbol{\%}$ & $\mathbf{n}$ & $\boldsymbol{\%}$ \\
\hline Obeso & 15 & 23,2 & 15 & 26,3 & 30 & 24,6 \\
Sobrepeso & 23 & 35,4 & 9 & 15,8 & 32 & 26,2 \\
Normal & 27 & 41,5 & 33 & 57,9 & 60 & 49,2 \\
Bajo peso & 0 & 0,0 & 0 & 0,0 & 0 & 0,0 \\
\hline
\end{tabular}

$23,1 \%$, esto es similar a lo encontrado para población escolar municipalizada de la IX región por Silva et al. (2005) y Silva et al. (2008), diferenciándose de lo encontrado por Martínez et al. en donde la obesidad promedio es mayor en los varones de la muestra.
La muestra presenta valores mayores en sobrepeso y obesidad un 50,8\% de, al comparar con un estudio nacional efectuado en escolares de $8^{\circ}$ básico de edades entre 13-15 años, de los cuales un 40,2\% de ellos presentaban sobrepeso u obesidad (MINEDUC). Estos antecedentes nos permiten 
MARTÍNEZ, C.; REINIKE, O.; SILVA, H.; CARRASCO, V.; COLLIPAL, E. \& JIMÉNEZ, C. Composición Corporal y Estado Nutricional de una Muestra de Estudiantes de 9 a 12 años de edad de Colegios Municipalizados de la Comuna de Padre las Casas, Región de la Araucanía-Chile. Int. J. Morphol., 31(2):425-431, 2013.

estimar que de mantenerse la prevalencia de la obesidad y el sobrepeso en los escolares de la Región de La Araucanía, estos sobrepasaran los resultados obtenidos en la encuesta de salud del año 2010, donde la prevalencia de exceso de peso en la población adulta es del 64,5\%, la cual corresponde al $39,32 \%$ de sobrepeso y un $25,13 \%$ de obesidad (MINSAL, 2011).

En un estudio de prevalencia realizado en Santiago, mostró que la epidemia de obesidad en Chile está afectando progresivamente a los preescolares de menor edad, dándose la paradoja, que junto con estar concluyendo la celebración de la erradicación de la desnutrición, se debe afrontar las consecuencias de la obesidad (Kain et al., 2007).

En Chile, como en todos los países del mundo ha habido procesos de transición nutricional, demográfica y epidemiológica Popkin (2001). El cambio nutricional está asociado a variables tales como el aumento de los ingresos y urbanización-modernización, ocio, trabajo, la influencia de los medios de comunicación de masas y el marketing de alimentos (Popkin, 1998, 2001; Rivera et al., 2002; Albala \& Vio, 2006).

Los cambios de la sociedad actual, el desarrollo de conductas y estilo de vida propio de las áreas urbanas e industrializadas donde predomina un ambiente Obesigénico con el consecuente aumento de los problemas de salud influenciados por el aumento de la obesidad desde etapas tempranas de desarrollo.

Comparando los resultados obtenidos por el método de fraccionamiento de Débora Kerr entre hombres y mujeres, apreciamos diferentes resultados en los cinco componentes $(\mathrm{p} \leq 0,05)$.

En el análisis por sexo de las muestra se encuentran diferencias estadísticamente significativas. En la MA a favor de las mujeres $(\mathrm{p}=0,01)$, en cambio los varones presentan diferencias estadísticamente en la MM con un valor de $(\mathrm{p}=0,003)$. En la comparación entre las edades de la muestra se presentan diferencias, con una significancia de $(\mathrm{p}=0,029)$ en la MA y un $(\mathrm{p}=0,001)$ en la MM, ambas para los 9, 10 y 11 años, los valores promedios de los porcentajes de MA, son un 27,03 en varones y un 29,76 en damas, valores superiores a un estudio realizado en población de sujetos físicamente activos de rango de edad 20 a 29 años, donde se encontraron porcentajes promedio de MA de 21,6 $\pm 4,1$

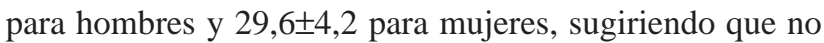
preocupa la diferencia entre ambos sexos, dada la conocida funcionalidad del tejido adiposo en mujeres, pero si el elevado porcentaje graso que compone la morfoestructura femenina de este grupo especifico (Rodríguez et al., 2010). Situación que es superada por los resultados encontrados para la muestra de la población escolar municipalizada de la comuna de Padre Las Casas.

MARTÍNEZ, C.; REINIKE, O.; SILVA, H.; CARRASCO, V.; COLLIPAL, E. \& JIMÉNEZ, C. Body composition and nutritional status of 9 to 12 year old Municipal schools students sample of Padre las Casas commune, Araucanía Region, Chile. Int. J. Morphol., 31(2):425-431, 2013.

SUMMARY: The study of Body Mass Index (BMI) is the standard recommended by the WHO for nutritional status according to the technical standard nutritional force in Chile. Anthropometric studies to estimate body composition study the morphology and dimensions. This study describes the nutritional situation and body composition of a sample of municipal schools of the municipality of Padre las Casas. We evaluated 122 adolescents of both sexes, 65 men (53.2\%) and 57 women (46.7\%), between 9 and 12 years old. For body composition was used anthropometric method described by Dhebora Kerr, while evaluation and classification of nutritional status was conducted according to technical rules nutritional Chilean Health Ministry. The men had a body composition dominated muscle mass (MM) on the other components, with values of $38.79 \pm 3.65 \%$ at 9 years of age, $40.80 \pm 3.36 \%$ at 10 years age, $41.47 \pm 2.52 \%$ at 11 years of age, $43.35 \pm 1.59 \%$ at 12 years of age. In women also predominate MM but lower figures, with a percentage of $38.70 \pm 6.64 \%$ at 9 years of age, $39.85 \pm 3.84 \%$ at 10 years of age, $39.56 \pm 4.41 \%$ at 11 years of age, $40.28 \pm 2.37 \%$ at 12 years of age. The nutritional status of the sample has the following results in the case of males $23.1 \%$ were obese and $35.4 \%$ were overweight. In womans $26.3 \%$ were obese and $15.8 \%$ were overweight. When comparing the results of Adipose Mass and MM were no significant differences by gender and ages of the sample with a value of $\mathrm{p} \leq 0.05$. The atmosphere obesigénico which highlights the lifestyle modification, diet and the effect of inactivity, is affecting the school population of Padre Las Casas.

KEYWORDS: Anthropometry; Body mass index; Nutritional state.

\section{REFERENCIAS BIBLIOGRÁFICAS}

Albala, C. \& Vio, F. Obesidad y pobreza: un desafio pendiente en Chile. In: Peña M, Bacallao J. La obesidad en la pobreza: un nuevo reto para la salud pública. Washington, D.C.: Organización Panamericana de la Salud, 2006. pp.46-56, 
MARTíneZ, C.; REINIKE, O.; SILVA, H.; CARRASCO, V.; COLLIPAL, E. \& JIMÉNEZ, C. Composición Corporal y Estado Nutricional de una Muestra de Estudiantes de 9 a 12 años de edad de Colegios Municipalizados de la Comuna de Padre las Casas, Región de la Araucanía-Chile. Int. J. Morphol., 31(2):425-431, 2013.

Alegría, E.; Cordero, A.; Laclaustra, M.; Grima, A.; León, M.; Casanovas, J. A.; Luengo, E.; Del Río, A.; Ferreira, I. Investigadores del registro MESYAS. Prevalencia del síndrome metabólico en población laboral española. El registro MESYAS. Rev. Esp. Cardiol.; 58:797-806, 2005.

Almagià, A. F.; Rodríguez, R. F. J.; Barraza, G. F. O.; Lizama, P. J.; Ivanovic, D. \& Binvignat, G. O. Perfil antropométrico de jugadores Profesionales de voleibol Sudamericano. Int. J. Morphol., 27(1):53-7, 2009.

Almagià, A. F.; Rodríguez, R. F. J.; Barraza, G. F. O.; Lizama, P. J. \& Jorquera, A. C. A. Perfil antropométrico de jugadoras Chilenas de futbol femenino. Int. J. Morphol., 26(4):817-21, 2008.

Álvero, J. R.; Cabañas, M. D.; Herrero de Lucas, A.; Martínez, L.; Moreno, C.; Porta, J.; et al. Protocolo de valoración de la composición corporal para el reconocimiento médico-deportivo. Documento de consenso del grupo español de cineantropometría de la federación española de medicina del deporte. Arch. Med. Deporte, 131:166-79, 2009.

Bibbins-Domingo, K.; Coxson, P.; Pletcher, M. J.; Lightwood, J. \& Goldman, L. Adolescent overweight and future adult coronary heart disease. N. Engl. J. Med., 357(23):2371-9, 2007.

Burrows, R. A.; Díaz, N. \& Muzzo, S. Variations of body mass index (BMI) according to degree of pubertal development. Rev. Med. Chil., 132(11):1363-8, 2004.

Carrasco, V.; Martinez, C.; Silva, H.; Collipal, E.; Campos, C. \& Silva, T. Prevalence of obesity in a sample of schoolchildren from municipalized schools in the IX Region of Chile 20082009. Int. J. Morphol., 29(3):830-4, 2011.

Carter J. E. L. \& Heath-Roll, B. H. Somatotyping: development and applications Cambridge, Cambridge University Press, 1990.

Clarys, J. P.; Martin, A. D. \& Drinkwater, D. T. Gross tissue weights in the human body by cadaver dissection. Hum. Biol., 56(3):459-73, 1984.

Cheng-Ye, J. P. \& Ohsawa, S. Changes in somatotype during growth in Chinese youth 7-18 years of Age. Am. J. Hum. Biol., 8(3):347-59, 1996.

da Silva, A. I. Somatotype and Physical Fitness of the Assistant Referees in Soccer. Int. J. Morphol., 29(3):792-98, 2011.

Del Sol, M. \& Henríquez, J. Evaluación de algunos índices antropométricos de un grupo de población mapuche. Bol. Mus. Araucano, 2:79-89, 1987.

Díaz, E.; Saavedra, C.; Lira, M., Bustos, E. \& Rivera, I. Evaluación de la condición física en adultos chilenos. Rev. Cienc. Act. Fís. Inst. Nac. Deportes, 3:7-13, 2008.
Espinoza, O.; Vega, C.; Urrutia, A.; Moreno, A. \& Rodríguez, H. Patrones antropométricos y consumo máximo de oxigeno (VO2) entre niños escolares chilenos aimaras y no aimaras de 10 a 12 años, que viven en altura $(3.500 \mathrm{~ms})$ y la planicie $(500$ ms). Int. J. Morphol., 27(4):1313-8, 2009.

Kain, J.; Lera, L.; Rojas, J. \& Uauy, R. Obesidad en preescolares de la Región Metropolitana de Chile. Rev. Med. Chil., 135(1):6370, 2007.

Kerr, D. A. An anthropometric method for the fractionation of skin, adipose, muscle, bone and residual tissue masses in males and females age 6 to 77 years. MSc. Thesis, Simon Fraser University, 1988.

Martínez, C.; Silva, H.; Collipal, E. \& Carrasco, V. Descripción del somatotipo e IMC en una muestra de adolecentes de colegios municipalizados de la ciudad de Temuco - Chile. Int. J. Morphol., 26(3):653-7, 2008.

Ministerio de Educación (MINEDUC). Informe de resultados de Educación Física SIMCE $8^{\circ}$ básico. Santiago, Unidad de Curriculum y Evaluación, SIMCE, 2010. Disponible en: http:// www.ind.cl/estudios-e-investigacion/investigaciones/ D o c u ments/2012/S i m c e _ Informe _ d e Resultados_Educacion_Fisica.pdf.pdf

Ministerio de Salud (MINSAL). La obesidad y el sobrepeso en Chile podrán afectar a más de nueve millones de personas en el año 2010. Santiago, Ministerio de Salud, 2003.

Ministerio de Salud (MINSAL). Norma Técnica de Evaluación Nutricional del niño de 6 a 18 años. Rev. Chil. Nutr., 31(2):12837, 2004.

Ministerio de Salud (MINSAL). Encuesta nacional de salud, 20092010. Santiago, Gobierno de Chile, Ministerio de Salud, 2011.

OMS. Nota descriptiva: Obesidad y Sobrepeso. 2009. Disponible en: http://www.who.int/topics/obesity/es/

Peña, M. \& Bacallao, J. Obesidad y pobreza un nuevo desafío de la salud pública. São Paulo, Roca, 2006.

Popkin, B. M. La transición de la nutrición y sus implicaciones en países mal pagados. Nutrición de Salud Pública, 5-21, 1998.

Popkin, B. M. La transición de la nutrición y obesidad en el mundo en desarrollo. Diario de nutrición, 871-3, 2001.

Rivera, J. A.; Barquera, S.; Campirano, F.; Campos, I.; Safdie, M. \& Tobar, V. Epidemiological and nutritional transition in Mexico: rapid increase of non- comunicable chronic diseases and obesit. Pubublic Health Nutr., 5(1A):123-22, 2002.

Rodríguez, F. J. R.; Flores, A. A. A.; Farías, T. Y.; Gutiérrez, O. B. \& Arce, P. L. Body Composition and Referential Somatotype of Physically Active Subjects. Int. J. Morphol., 28(4):1159-65, 2010. 
MARTÍNEZ, C.; REINIKE, O.; SILVA, H.; CARRASCO, V.; COLLIPAL, E. \& JIMÉNEZ, C. Composición Corporal y Estado Nutricional de una Muestra de Estudiantes de 9 a 12 años de edad de Colegios Municipalizados de la Comuna de Padre las Casas, Región de la Araucanía-Chile. Int. J. Morphol., 31(2):425-431, 2013.

Sánchez-Muñoz, C.; Sanz, D. \& Zabala, M. Anthropometric characteristics, body composition and somatotype of elite junior tennis players. Br. J. Sports Med., 41(11):793-9, 2007.

Silva, H.; Bruneau, J.; Reyno, P. \& Bucarey, S. Somatotipo e índice de masa corporal en una muestra de adolescentes de ambos sexos de la ciudad de Temuco, Chile. Int. J. Morphol., 21(4):309-13, 2003.

Silva, H.; Collipal, E.; Martínez, C. \& Bruneau, J. Evaluación de los Componentes del Somatotipo e Índice de Masa Corporal en Escolares del Sector Precordillerano de la IX Región, Chile. Int. J. Morphol., 23(2):195-9, 2005.

Silva, H.; Collipal, E.; Martínez, C. \& Torres, I. Análisis del IMC y Somatotipo en una muestra de adolescentes con sobrepeso y obesidad en Temuco- Chile. Int. J. Morphol., 26(3):707-11, 2008.

Sociedad Internacional para el Avance de la Kineantropometría (ISAK). Estándares internacionales para la valoración antropométrica. España, Universidad Católica de San Antonio de Murcia-Sociedad Internacional para el Avance de la Kinantropometría, 2001.

Toro, T. \& Almagià, A. Aplicación de estándares antropométricos para la evaluación del crecimiento y estado nutricional en niñas y niños de 11-15 años de Valparaíso. An. Anat. Nor., 7:99107, 1989.

\author{
Dirección para correspondencia \\ Prof. Cristian Martínez Salazar \\ Departamento de Educación Física \\ Universidad de la Frontera \\ Casilla 54 D \\ Temuco \\ CHILE
}

Email: cmartin@ufro.cl

Recibido : 28-11-2012

Aceptado: 05-04-2013 